\title{
Pulmonary arterial hypertension: combination therapy in the modern management era
}

\section{To the Editor:}

I read with interest the recent review article by GALIE et al. [1] on the use of combination therapy in pulmonary arterial hypertension (PAH). The authors raised a number of important points, including the use of a goal-oriented approach to combination therapy and the need for regular monitoring in order to achieve optimal results for an individual patient.

In support of this approach, I would like to present the case of a 40-yr-old female, who had complained of progressive dyspnoea on exertion during a third pregnancy, becoming particularly noticeable after delivery in March 2005. Her two previous pregnancies had been uneventful and she had no relevant previous medical history. She was initially diagnosed as suffering from fatigue and post-partum depression in August 2005.

The patient was eventually referred to a cardiologist in October 2005, where she was classified as being in New York Heart Association/World Health Organization Functional Class (NYHA/WHO FC) III, with a resting heart rate of 90 beats. $\min ^{-1}$ and right axis deviation on ECG. Transthoracic echo/ Doppler showed the right heart to be dilated, with a tricuspid regurgitation velocity of $4.7 \mathrm{~m} \cdot \mathrm{s}^{-1}$ (estimated right ventricular systolic pressure $100 \mathrm{mmHg}$ ) and no evidence of congenital heart disease.

When the patient was subsequently referred to our specialist centre in November 2005, she had deteriorated clinically since her initial diagnosis 3 months previously, and was suffering from chest pain and dizziness. There was no evidence of connective tissue disease and she was HIV negative. There was also no evidence of pulmonary thromboembolism on ventilation/perfusion lung scan, or of portal hypertension on abdominal ultrasonography. During exercise testing, her 6min walk distance (6MWD) was $519 \mathrm{~m}$ (79\% of predicted value), her Borg dyspnoea score was 6 , her heart rate increased from 78 to 82 beats $\cdot \mathrm{min}^{-1}$ and her oxygen saturation reduced from $96 \%$ to $79 \%$. On right heart catheterisation, the patient was found to have pulmonary hypertension (mean pulmonary arterial pressure $55 \mathrm{mmHg}$ ), low cardiac index (CI; $2.01 \mathrm{~L} \cdot \mathrm{min}^{-1} \cdot \mathrm{m}^{-2}$ ) and high pulmonary vascular resistance $\left(1,248 \mathrm{dyn} \cdot \mathrm{s} \cdot \mathrm{cm}^{-5}\right)$, and was nonvasoreactive after inhalation of nitric oxide. Based on these findings she was diagnosed with idiopathic PAH NYHA/WHO FC III and treatment with bosentan was started. The goal of therapy was to bring this patient into NYHA/WHO FC II.

At reassessment 4 months after initiation of therapy, her condition was relatively stable, with a similar $6 \mathrm{MWD}$ and right atrial pressure $(P$ ra $)$ but improved CI $\left(2.50 \mathrm{~L} \cdot \mathrm{min}^{-1} \cdot \mathrm{m}^{-2}\right)$. However, as she remained in NYHA/WHO FC III and had elevated levels of brain natriuretic protein (BNP; $\left.217 \mathrm{pg} \cdot \mathrm{mL}^{-1}\right)$, the decision was made to escalate treatment by adding sildenafil on to existing bosentan therapy. On further reassessment after 4 months of combination therapy, the patient's condition was found to have worsened. She remained in NYHA/WHO FC III, but had a decreased 6MWD $(441 \mathrm{~m})$, decreased CI $\left(2.09 \mathrm{~L} \cdot \mathrm{min}^{-1} \cdot \mathrm{m}^{-2}\right)$ and increased BNP $\left(360 \mathrm{pg} \cdot \mathrm{mL}^{-1}\right)$. Epoprostenol was therefore added to bosentan and sildenafil. After 4 months of triple therapy, the patient had improved to NYHA/WHO FC II and all major parameters were improved (6MWD $601 \mathrm{~m}$, Pra $3 \mathrm{mmHg}, \mathrm{CI}$ $3.35 \mathrm{~L} \cdot \mathrm{min}^{-1} \cdot \mathrm{m}^{-2}$ and $\left.\mathrm{BNP} 62 \mathrm{pg} \cdot \mathrm{mL}^{-1}\right)$. At reassessment after a further year, the patient was stable in NYHA/WHO FC II with sustained haemodynamic improvement.

The successful management of this patient supports a number of points discussed by GALIÈ et al. [1] in their review article. In particular, a goal-oriented approach to treatment and regular reassessment against a range of pre-defined parameters were central to the success of combination therapy in this case. Their findings, together with this case study and others [2], also demonstrate how the recently published treatment guidelines of the European Society of Cardiology (ESC) and the European Respiratory Society (ERS) [3] can be implemented to improve patient outcomes.

The recommendations from ESC/ERS outline how a set of parameters known to be of prognostic importance in $\mathrm{PAH}$ can be used to distinguish between patients with a "better prognosis" and those with a "worse prognosis". In this case, our patient was found to be relatively stable at the first assessment following treatment initiation, although she showed no signs of clinical improvement. Whereas previous guidelines have advocated a step-up approach to therapy only in cases of worsening $\mathrm{PAH}$, the latest recommendations suggest that the goal of therapy today should be improvement of patients in NYHA/WHO FC III-IV to NYHA/WHO FC I-II [4]. In our case, therefore, the patient was deemed to be stable but the treatment response was unsatisfactory; as such, consideration of treatment modification was required.

The importance of regular assessment after changes in treatment was also clearly demonstrated in our case by the fact that her condition had worsened 4 months later, with several parameters being indicative of a "worse prognosis" according to the ESC guidelines, classifying her as "unstable and deteriorating" and in need of immediate attention. Assessment 4 months after the initiation of triple therapy showed that the majority of the patient's parameters were improved to the point that they were in the "better prognosis" category, and the patient was in a "stable and satisfactory" condition. 
Finally, this case demonstrates the importance of timely and correct diagnosis in PAH. Evidence suggests that treatment of mildly symptomatic patients can be beneficial, and indeed data from the EARLY trial [5] led to regulatory authority approval for the use of bosentan in the treatment of $\mathrm{PAH}$ patients in NYHA/WHO FC II and a recommendation for treatment of such patients in recent guidelines [3]. In this case the patient's condition deteriorated between her initial misdiagnosis of post-partum depression and her referral to our specialist centre 3 months later, removing the option for early treatment before her symptoms became marked.

\section{O. Sitbon}

Université Paris Sud-11, Centre de Référence de l'Hypertension Pulmonaire Sévère, Service de Pneumologie, INSERM U999, Hôpital Antoine Béclère, Clamart, France.

Correspondence: O. Sitbon, Service de Pneumologie, Hôpital Antoine Béclère, 92141, Clamart, France. E-mail: olivier.sitbon@ abc.aphp.fr

Statement of Interest: O. Sitbon has relationships with drug companies including Actelion, Bayer-Schering, GSK, Lilly, Pfizer and United Therapeutics. In addition to being an investigator in trials involving these companies, relationships include consultancy service and membership of scientific advisory boards. He has received reimbursement for attending symposium and funds for research from Actelion, Pfizer, GSK, Lilly and Bayer-Schering. He has received fees for speaking from Actelion, Bayer-Schering, GSK, Lilly, Pfizer and United Therapeutics.

Provenance: Publication of this peer-reviewed article was supported by Actelion Pharmaceuticals Ltd, Switzerland (unrestricted grant, European Respiratory Review issue 118).

Acknowledgements: Editorial assistance was frovided by L. Thomas, Elements Communications Ltd (Westerham, UK), supported by Actelion Pharmaceuticals Ltd (Allschwil, Switzerland).

\section{REFERENCES}

1 Galiè N, Negro L, Simonneau G. The use of combination therapy in pulmonary arterial hypertension: new developments. Eur Respir Rev 2009; 18: 148-153.

2 Montani D, O'Callaghan DS, Jaiis X, et al. Implementing the ESC/ ERS pulmonary hypertension guidelines: real-life cases from a national referral centre. Eur Respir Rev 2009; 18: 272-290.

3 Galiè N, Hoeper MM, Humbert M, et al. Guidelines for the diagnosis and treatment of pulmonary hypertension. Eur Respir J 2009; 34: 1219-1263.

4 Barst RJ, Gibbs JS, Ghofrani HA, et al. Updated evidence-based treatment algorithm in pulmonary arterial hypertension. J Am Coll Cardiol 2009; 54: Suppl. 1, S78-S84.

5 Galiè N, Rubin LJ, Hoeper M, et al. Treatment of patients with mildly symptomatic pulmonary arterial hypertension with bosentan (EARLY study): a double-blind, randomised controlled trial. Lancet 2008; 371: 2093-2100.

\section{Association of pulmonary aspergilloma and allergic bronchopulmonary aspergillosis}

\section{To the Editor:}

We have read with interest recent reports on the complex interactions between Aspergillus fumigatus and the lungs [1-3]. A. fumigatus may be involved in different respiratory diseases including aspergilloma, allergic bronchopulmonary aspergillosis (ABPA) or invasive infectious manifestations. However, these different manifestations are usually not concomitantly present and there are only a few studies that report the association between these complications [4-14]. We wish to present a case of fully documented aspergilloma and ABPA in a patient with difficult asthma, which represented a difficult therapeutic challenge.

A 50-yr-old female, nonsmoker was followed up since 2000 for difficult to control non-atopic asthma associated with severe rhinosinusitis. In March 2008, the patient presented to the respiratory department (Hôpital Antonie Béclère, Clamart, France) with a 1-month history of mild recurrent haemoptysis. Physical examination was normal. Chest radiography showed excavated lung opacity in the superior left field. A thoracic computed tomography $(\mathrm{CT})$ scan revealed a cavity with a fungus ball in the left upper lobe and two other opacities in the left lower lobe (fig. 1). There was no fungus ball in the sinuses. Fiberoptic bronchoscopy showed no abnormality. Bronchial fluid aspiration and bronchoalveolar lavage stains and cultures did not detect either resistant acid alcohol bacilli or Aspergillus. The eosinophil count was $1.5 \times 10^{9} \cdot \mathrm{L}^{-1}$ and total serum immunoglobulin (Ig)E levels were elevated $\left(2,236 \mathrm{KU} \cdot \mathrm{L}^{-1}\right.$, normal range: 0 $\left.113 \mathrm{KU} \cdot \mathrm{L}^{-1}\right)$. A fumigatus specific $\mathrm{IgE}$ was positive $\left(41.2 \mathrm{KU} \cdot \mathrm{L}^{-1}\right)$. Aspergillus serology including Aspergillus immunoelectrophoresis was positive. Antinuclear antibodies and antineutrophil cytoplasmic antibodies were negative. A CT scan of the chest revealed persistence of the left upper lobe opacity, disappearance of the other opacities and localised bronchiectasis of the left lower lobe. No evidence of transient pulmonary infiltrates has been documented. A diagnosis of concomitant aspergilloma and ABPA was suspected. A wedge resection of the left upper lobe was performed (fig. 2). The histopathological examination of the resected wedge revealed an aspergilloma with fungal hyphae (fig. 3). Culture of this material yielded A. fumigatus. The patient received itraconazole post-operatively at a dose of $300 \mathrm{mg} \cdot$ day $^{-1}$ and was continued on inhaled corticosteroids. 5 months after the intervention, her total serum IgE count dropped to $1,769 \mathrm{KU} \cdot \mathrm{L}^{-1}$ and her A. fumigatus specific IgE level dropped to $33.8 \mathrm{KU} \cdot \mathrm{L}^{-1}$. 